\title{
LA SITUATION DE LA CHANSON FRANCOPHONE EN PÉRIODE DE PANDÉMIE: ÉTAT DES LIEUX, RÉFLEXIONS ET IMPACT DANS L'APPRENTISSAGE/ENSEIGNEMENT DU FLE ${ }^{1}$
}

\begin{abstract}
ABRÉGÉ: Nous vivons tous dans une nouvelle ère façonnée par la pandémie et la propagation de la COVID-19. Par conséquent de nouvelles règles et de nombreuses restrictions se sont vues imposées dans tous les domaines du quotidien. Ainsi, la musique, mais aussi la chanson ont été impactées. Dans notre travail nous allons nous concentrer sur un corpus de 17 chansons francophones qui ont eu un écho via Internet. Nous allons montrer quel impact elles ont et comment elles pourront être utilisées en classe de FLE. En se basant sur la méthodologie analytique et comparative, nous allons montrer quels sont les avantages de leur utilisation en classe de FLE et ce qu'elles apportent au niveau technique, socioculturel et linguistique. Nous sommes d'avis qu'il revient aux enseignants de suivre chaque changement socioculturel, de l'adapter et de l'intégrer davantage non seulement dans l'apprentissage/enseignement des langues, mais aussi dans l'apprentissage/enseignement en général.
\end{abstract}

Mots clés: pandémie, chanson, français, francophonie, technologie, socioculturalité, linguistique, enseignement/apprentissage du FLE (français langue étrangère).

\section{FRANCOPHONE SONGS IN THE PANDEMIC: REFLECTIONS ON THE STATE OF ART AND THE IMPACT ON LEARNING/TEACHING FFL}

ABSTRACT: We are all living in a new era of humanity imposed by the spread of the COVID-19 pandemic. As a result, new rules and many restrictions have been put in place, affecting all areas of life, including music and songs. In our paper, we will be analyzing a corpus of 17 French songs that echoed throughout the Internet, in order to show what impact they have had on humanity and how they can be used in the French language classes. Based on analytical and comparative methodology, we will show what the advantages of their use in FFL classes are and what they introduce at the technical, socio-cultural and linguistic levels. We believe that it is up to teachers to

1 Cet article est rédigé dans le cadre du projet scientifique (1001-13-01) financé partiellement par l'Agence universitaire de la francophonie et l'Ambassade de France en Serbie. 
follow each socio-cultural change, adapt and integrate it further, not only in language learning/teaching, but also in learning/teaching in general.

Key words: pandemic, song, French, Francophonie, technology, socioculturality, linguistics, teaching/learning of FFL (French as a foreign language).

\section{INTRODUCTION}

Cela fait plus d'un an que l'on s'adapte à une nouvelle réalité et aux nouvelles circonstances de la vie. La COVID-19 et la pandémie nous ont obligés à suivre de nouvelles règles de comportement, qui dans un sens ont censuré certains actes socioculturels habituels, très importants pour la survie et la cohésion des êtres humains. Le port du masque, les gestes barrières, la distance physique, le couvrefeu, la fermeture des lieux publics et culturels, ne sont que quelques-uns des nouveaux phénomènes qui ont dangereusement aggravé la condition humaine. L'art en général, ainsi que les artistes, font partie de ceux qui ont été les plus touchés, car ils ont perdu leur place sur scène dans le monde entier.

C'est justement la raison pour laquelle dans le travail ci-présent nous allons nous concentrer sur l'un des domaines artistiques, la musique, et plus précisément, la chanson francophone dans un contexte de pandémie. D'après Yves Charles Zarka «l'être humain a toujours chanté. Cette affirmation n'est évidemment ni physique, ni historique mais métaphysique. Elle veut dire qu'il appartient à l'être de l'homme de chanter, tout autant qu'il appartient à son être ou à son essence de parler » (Zarka 2004: 4).

Si nous gardons en vue notre contemporanéité, nous pouvons dire que depuis l'apparition d'Internet, surtout des plateformes comme YouTube et d'autres réseaux sociaux, la chanson est devenue accessible à tous. Aujourd'hui, où l'industrie musicale est digitalisée, et où ses artistes ne peuvent plus se produire sur scène comme avant, la chanson a trouvé un autre biais pour se manifester. Grâce aux réseaux sociaux, elle circule très rapidement, surtout si elle est de qualité, elle dispose d'un accès rapide et instantané partout dans le monde. Parfois elle nous touche au plus profond du cœur, parfois elle passe inaperçue, ou alors on l'écoute en tant que simple divertissement. «Aujourd'hui, les chansons circulent très rapidement d'un pays à l'autre, et ainsi elles relient tous les coins du monde francophone ». (Manić-Matić, Popović 2012: 355) En ce qui concerne son rôle durant la pandémie nous trouvons que son statut a changé et qu'elle est aussi devenue un art par excellence, car nous pouvons voir beaucoup de nouveaux artistes et une nouvelle scène que nous n'avons pas connus auparavant. 
Pour pouvoir mieux analyser notre corpus, nous allons le présenter à travers trois aspects, c'est-à-dire nous allons voir ce que les chansons francophones nous apportent en temps de pandémie d'un point de vue technologique, socioculturel, linguistique, puis nous aborderons leur utilisation dans l'apprentissage/enseignement du FLE.

\section{ASPECT TECHNOLOGIQUE}

Lorsque qu'on mentionne des clips vidéo, nous pouvons dire que toutes les chansons choisies sont favorables pour le travail dans l'apprentissage/enseignement du FLE. La première raison de ce choix est que tout notre corpus est facilement accessible. Étant donné que tout le monde aujourd'hui est hyper connecté grâce à Internet, et fait partie de nombreux réseaux sociaux (YouTube, FaceBook, Instagram, Twitter, Flash Infos, etc.) via les ordinateurs, les portables, les smartphones, les tablettes, il est tout à fait naturel d'exploiter ces nouveaux supports dans le contexte de FLE. C'est pourquoi notre corpus est majoritairement issu de la plateforme YouTube, mais nous pouvons aussi le voir circuler via d'autres réseaux sociaux.

La deuxième raison importante de ce choix est la durée d'une chanson, qui en général dépasse rarement 5 minutes (sauf les chansons № 14, 15 et 16). Ensuite, le corpus choisi assure une bonne qualité de l'image et du son. En ce qui concerne l'image, les utilisateurs d'Internet font partie du public visuel, c'est-à-dire qu'ils ont plutôt tendance à regarder une chanson, parce que c'est l'image qui va les attirer, ainsi que son nombre de vues. Le son est aussi important, mais reste cependant au second plan par rapport à l'époque de la digitalisation de la musique. Aujourd'hui c'est l'image qui capte d'abord le public, ensuite, le son. "Les enregistrements audiovisuels constituent un support particulièrement riche et attrayant pour le cours de FLES ${ }^{2}$. Les apprenants, surtout les plus faibles d'entre eux, y trouvent une aide visuelle aux activités de compréhension orale ». (Defays 2018: 307)

Pour ce qui concerne notre choix, nous nous sommes aperçue que certains artistes avaient fait preuve de plus de créativité que d'autres. Dans certains clips vidéo nous pouvons voir plusieurs images d'un même endroit (chanson № 1 et 4 un appartement ; chanson № 11 et 13 - une maison). Parfois, on se déplace d'un continent à l'autre (chanson № 17), d'une ville à une autre (chanson № 6, 17), ou

\footnotetext{
${ }^{2}$ FLES - Français lange étrangère et seconde.
} 
encore on se déplace d'un endroit à l'autre dans une même ville (chanson № 12). Certains artistes sont moins créatifs et ne disposent que d'un seul cadre d'image (chansons № 2 et 5 - une pièce ; chanson № 3 - un studio), ou encore d'une image scindée en vignettes et où se trouvent les chanteurs ou les solistes qui sont bien mis en avant (chanson № 7 et 14). Parfois, on peut aussi voir un mélange de périodes, la période d'avant et celle d'après la pandémie (chanson № 15), ou encore un grand espace avec beaucoup de cadres (chanson №16 - Gare du Nord, Paris). Pour ce qui est des moyens technologiques, les chansons précédemment mentionnées ont été réalisées, soit à l'aide d'une simple caméra soit avec une caméra de téléphone portable (chansons № 2, 4, 5, 7 et 13), ou de plusieurs caméras de plusieurs participants (chansons № 1, 3, 6, 11, 16 et 17). La technologie de la plateforme Zoom a aussi bien été utilisée et combinée, ou encore celle de simples enregistrements vidéo qui ont été copiés, collés et intégrés dans une même vidéo (chansons № 6, 7,14 et 17). Il y avait parfois même des montages d'anciennes vidéos intégrées dans une chanson (chanson № 15).

Pourtant, il y a aussi d'autres moyens avec lesquels les chansons ont été réalisées. Il s’agit des exemples des chansons № 8, 9 et 10, qui sont en effet des reprises des dessins animés bien connus de la production «Walt Disney » (№ 8 «Le Livre de la Jungle », № 9 «La Petite Sirène » et № 10 « La Belle et la Bête »). Dans ces cas-là un autre procédé est en jeu, c'est-à-dire, une adaptation des personnages principaux des dessins animés chantant sur l'air de mélodies très connues de nouvelles paroles qui concernent la pandémie. De même, en parlant de son et d'airs connus, les auteurs des chansons № 1, 2, 4, 5, 11, 12 et 14 se sont aussi inspirés de mélodies célèbres francophones et anglophones (№ 1 Jacques Brel « Vesoul », № 2 Tex Lecor «Le Frigidaire », № 4 Lewis Capaldi «Someone you loved », № 5 comptine française «Lundi matin », № 8 la chanson du dessin animé de Disney Le Livre de la Jungle «Il en faut peu pour être heureux », № 9 la chanson du dessin animé de Disney, La Petite Sirène «Sous l'océan », № 10 la chanson du dessin animé de Disney, La Belle et la Bête «C'est la fête », № 11 Les Cowboys Fringants «L’Amérique Pleure du groupe », № 12 Georges Brassens «Les amoureux des bancs publics », № 14 Michael Jackson « We are the world »).

Lorsque nous visionnons un clip sur la plateforme YouTube, nous ne regardons ni n'entendons pas seulement la chanson choisie, mais nous avons accès à d'autres informations importantes. Cette plateforme nous donne aussi l'information via ses outils numériques, combien de fois une chanson a été vue/entendue, etc. Nous pouvons y voir également si elle est populaire ou non. De plus, YouTube assure une interaction virtuelle grâce à ses outils, où chacun peut 
laisser son commentaire à propos d'un clip de la chanson et dire aussi si elle a été aimée, «likée », ou non, ou encore peut laisser d'autres commentaires. D'après tout ce qui précède nous pouvons dire que toutes les chansons du corpus assurent un bon son et une bonne image, donc elles peuvent être bien utilisées en tant que supports pédagogiques dans l'apprentissage/enseignement du FLE.

\section{ASPECT SOCIOCULTUREL}

«La chanson dit donc beaucoup plus de choses qu'on ne croit, beaucoup plus de choses qu'on n'en perçoit immédiatement sur les êtres, les passions et les rapports sociaux » (Zarka 2004: 4). La chanson surtout en pandémie représente un phénomène social particulier et tout à fait réaliste. Elle reflète bien les nouvelles circonstances en pandémie, le nouvel entourage, ainsi que l'état psychique et physique des gens confinés. Les apprenants «y découvrent aussi les éléments culturels qui leur manquent si le cours ne se déroule pas dans un pays francophone : les conditions proxémiques (la distance entre les interlocuteurs), les attitudes corporelles, les expressions du visage, (...)» (Defays 2018: 307-308).

Les artistes des chansons concernées sont issus de milieux socioculturels variés francophones, de tout âge, plus ou moins connus dans le monde francophone. Ce qui représente un atout d'Internet c'est que chaque personne qui s'y connait en informatique et qui est talentueuse, qui sait chanter et produire de la musique, peut se promouvoir grâce à un accès facile aux réseaux sociaux. Dans notre cas il s'agit de chansons destinées à toutes les générations où tout le monde peut s'identifier. Elles dévoilent bien un monde francophone avec toutes ses variétés (France (chansons № 1, 2, 3, 5, 6, 13, 15, 16 et 17), Suisse (chanson № 12), Canada francophone (chansons № 4, 11 et 14), Afrique francophone - le Maroc (chanson № 7), Belgique (chanson № 17), La Réunion (chanson № 17), Caraïbes (chanson № 17)). Parfois, elles dépassent le monde francophone, lorsque quelques strophes sont chantées en italien et en espagnol (chansons № 6 et 17). De plus, elles sont parfois traduites en anglais, pour être potentiellement diffusées dans le monde entier (chanson № 6) et pour montrer qu'en pandémie, on souffre de la même manière. Nous pouvons voir que toutes les cultures via la chanson critiquent, se moquent, râlent, ont de la peine, souffrent ou rient de la nouvelle situation (Symphonie confinée «La tendresse », chansons № 6 et 7). Dans ces derniers exemples, l'Autre n'existe presque pas, ainsi que l'interculturalité, car on devient tous égaux dans cette crise sanitaire globale. Ici, il s'agit d'un atout dans l'acceptation et la valorisation de la culture des autres et de la sienne, surtout en parlant des apprenants serbophones, car les frontières entre les cultures dans la 
pandémie dans un sens s'effacent et nous vivons tous pareillement. Il faudrait aussi mentionner que dans l'apprentissage des langues étrangères dans les Balkans, ce fait est très important car dans notre pays les cultures étrangères étaient surtout représentées au détriment des cultures balkaniques, comme de grandes civilisations colonisatrices, où on ressentait une certaine dévalorisation de la culture maternelle. Avec la pandémie ces différences se perdent et le monde entier est uni contre la COVID-19.

Toutes les chansons en pandémie sont réalistes et authentiques, ce qui est un autre atout pour l'apprentissage/enseignement du FLE. Elles deviennent sociales, voire activistes et engagées (chansons № 1, 2, 6, 7, 14, 15, 16 et 17), parfois bienfaisantes avec leur humour parodique (chansons № 3, 4, 5, 11, 12 et 13), et même ludiques, grâce à leurs formes enfantines (chansons № 8, 9 et 10).

En plus de cela nous pouvons voir toute la France et la francophonie de manière plus authentique, dans leur domicile ou dans des lieux publics (gares, salons, marchés, jardins, toits des maisons et immeubles, mers, écoles de dance, monastères, écoles, patinoires, etc.). De même, le code vestimentaire des gens qui sont dans les vidéos, où on voit tout d'abord le port des masques et une nonchalance dans l'habillement du quotidien, est un bon exemple culturel surtout pour les jeunes apprenants serbophones. Étant donné qu'ils sont majoritairement influencés par la culture américaine via la musique et les films, culture portée grandement sur l'apparence, parfois même d'une manière exagérée, ils ont justement dans ces exemples l'occasion de voir des gens ordinaires qui revendiquent des conditions et des valeurs saines pour vivre.

Les chansons № 1, 3, 4, 5, 11 et 12 sont des parodies où l'on se moque du destin de l'humain en confinement, où l'on critique les politiciens et les nouvelles règles de vie (les gens enfermés dans leurs domiciles, la possession des attestations pour pouvoir sortir, les gestes barrières, le guide à travers la maison, les artistes sans public, etc.). La Serbie à la différence des autres pays francophones avait connu seulement une période de confinement rigide, ce qui n'était pas le cas de la France qui a été confinée trois fois et où les gens avaient besoin d'attestations pour pouvoir circuler en ville. Il s'agit d'un rare phénomène socioculturel que les apprenants serbophones vont rencontrer dans l'exemple de la chanson № 3, et qui n'est pas en vigueur dans notre pays. De même, en exploitant certaines chansons les apprenants peuvent aussi en apprendre plus sur la scène politique francophone, sur les noms de politiciens importants (chansons № 1, 3, 4 et 5). Par ailleurs, il y en a d'autres de notre corpus figurant dans certains médias (chanson № 3 diffusée par «TV Paris Première » et chanson № 17 diffusée par la chaîne du journal 
«L'Humanité »), qui ont pour objectif de rendre les gens socialement responsables, par exemple de porter le masque, de tenir une distance physique, parfois invitant à s'unir et à réagir pour une humanité plus radieuse.

Pour ce qui est des autres comportements, dans ces chansons nous pouvons voir les gens seuls confinés (chansons № 1, 2, 4, 5, 11 et 13), ou une image des gens dans une révolte douce où on revendique une liberté, une vie sociale sans distance physique où on va chanter et danser comme avant (chansons № 15, 16 et 17). En effet, dans ce dernier exemple (chansons № 15, 16 et 17), il s'agit d'une même chanson et de ses reprises. Sur les différents réseaux elle est devenue virale et prend une forme presque hymnique, pour des gens qui chantent les droits de l'homme en tant qu'êtres sociaux et citoyens du monde.

« Cette chanson de HK et ses amis Saltimbanks est devenu un véritable hymne à la défense de la culture et de l'art. Le cri de ralliement des artistes qui occupent les théâtres de France depuis plusieurs mois, mouvement qui s'était formé pour protester contre la fermeture des lieux culturels à cause de la pandémie de Covid19. » (source Internet de La Presse de la Manche consultée le 6 juillet 2021)

C'est pour cela qu'elle a été traduite en espagnol, italien, allemand (№ 17), et elle a aussi une reprise où les sous-titres sont en anglais ${ }^{4}$, c'est-à-dire que l'auteur de cette chanson voulait attirer au maximum l'attention du public partout dans le monde, tout en réclamant la vie que nous avons tous vécue avant la pandémie. Cette constatation nous allons la justifier par le fait que le chanteur de la chanson «Danser encore », Kaddour Hadadi ${ }^{5}$, est déjà connu sur la scène francophone comme une personne activiste, très soucieuse de l'humanité et pour de nombreux sujets en France. De même, ce n'est pas seulement une «chanson », mais « la chanson internationale en pandémie », où ce n'est pas seulement toute la France qui chante, comme si c'était la Marseillaise, mais bien au-delà, les peuples de toutes les nationalités.

\footnotetext{
3 https://actu.fr/normandie/cherbourg-en-cotentin_50129/le-chanteur-hk-et-son-spectaclerevendicatif-danser-encore-bientot-a-cherbourg_42103558.html?utm_medium=Social\& utm_source=Facebook\#Echobox $=1622112695$

${ }^{4}$ Nous n'avons pas mis dans notre corpus cet exemple pour ne pas l'encombrer, mais il est bien disponible sur le lien suivant : https://www.youtube.com/watch?v=g3NT0cirYh0

${ }^{5}$ Sur le lien suivant de la RFI (Radio France internationale) nous pouvons trouver une certaine biographie sur le chanteur Kaddour Hadadi : https://www.facebook.com/RFI/ videos/159376889330430
} 


\subsection{L'esthétique musicale}

«L'ancienne musique est liée à la poésie; or la nouvelle musique ne tente pas de transférer ce que nous portons en nous-mêmes dans le monde réel, mais c'est l'extérieur qui se reflète sur la vie intérieure (non comme une réalité, mais comme une possibilité de la réalité) $»^{6}$ (Knežević 2011: 75).

Justement, c'est ce que les chansons de notre corpus essayent de transmettre. À travers une esthétique musicale et visuelle les auteurs des chansons essayent en effet de sortir d'eux-mêmes la souffrance et le mécontentement qu'on vit dans la pandémie et à travers la musique ils cherchent une sorte de refuge et d'apaisement de leurs chagrins. Avec ces clips vidéo on démontre une nouvelle esthétique de la mélodie, des instruments et des voix de toutes les générations. La voix au premier plan, en tant qu'instrument suprême, comme dans les églises, est légèrement accompagnée avec des instruments variés. Les artistes talentueux et ceux moins talentueux représentent en effet la voix du peuple, de tous les citoyens du monde. Il est très important de montrer cette nouvelle esthétique musicale dans notre contexte de l'apprentissage/enseignement du FLE, car ces vingt dernières années la musique contemporaine en Serbie a connu une certaine décadence notamment avec les chaînes TV PINK et la musique turbo-folk. Il s'agit d'un phénomène musical et socioculturel qui a prédominé sur toutes les scènes et a mis malheureusement en arrière-plan une bonne et belle esthétique musicale.

Pour ce qui est des instruments dans le corpus choisi, nous pouvons en voir une large palette. L'instrument le plus représenté est la guitare, mais c'est tout à fait normal et attendu, car c'est un instrument très accessible et très populaire partout. Cependant, nous pouvons dire qu'elle est au premier plan dans les chansons № 5, 11 et 12. Dans la chanson № 5 nous avons une reprise de la comptine «Lundi matin », d'Alex Fredo, jeune chanteur et humoriste du Canada francophone. C'est une représentation ludique de la chanson, et vu le fait que l'original est destiné aux enfants, il est tout à fait justifié qu'elle soit présentée au son de la guitare, comme la plupart des comptines. La présence de la guitare dans la chanson № 11, du groupe Cowboys Fringants, en effet fait partie de l'identité québécoise et de la musique pop-country au Canada francophone. Pour ce qui est de la chanson № 12, c’est une reprise de la chanson du fameux chanteur français

\footnotetext{
${ }^{6}$ Citation originale : «Stara muzika je vezana uz poeziju; dok nova ne teži izlaganju onoga iz nas u realan svet, već spoljašnje ogleda u unutrašnjem životu (ne kao stvarnost, već kao celokupnu mogućnost realnosti).» (Knežević 2011 : 75)
} 
Georges Brassens par des artistes suisses, dont presque toutes les chansons sont accompagnées de guitare.

Le deuxième instrument, et non pas le moins important, est l'accordéon, un instrument traditionnel en France. Dans certaines chansons sa présence est même stéréotypée et accentuée (chanson № 3), et parfois il constitue un accompagnement naturel, une sorte de symbole folklorique de la musique traditionnelle française (chanson № 15). Il s’agit ici de bons exemples de la motivation en classe de FLE, car les apprenants serbophones pourront s'y identifier, étant donné que l'accordéon est un point commun entre les deux pays, la France et la Serbie, en ce qui concerne les instruments traditionnels. De plus, comme il est souvent dévalorisé en Serbie, car très utilisé dans les danses folkloriques, dans les chansons choisies ici, il change de statut et accompagne la chanson contemporaine et moderne.

Dans notre corpus nous avons aussi des chansons qui représentent des reprises d'airs connus. Pour attirer plus facilement leur public, les artistes de ces chansons se sont servis d'artistes déjà célèbres et valorisés (Tex Lecor (chanson № 2), Georges Brassens (chanson № 12), W. Disney (chansons № 8, 9 et 10), comptine (chanson № 5)). De même, en s'appuyant sur ces mélodies connues il y a plus de chance que le public soit attiré et séduit, car il les connait déjà et il va pouvoir s'y identifier plus vite.

\subsection{L'esthétique visuelle}

Pour ce qui est de l'esthétique visuelle, ces chansons la changent considérablement. Étant donné que l'objectif de leurs auteurs est de transmettre un message, ils ne se sont pas trop souciés de la représentation de l'image et parfois elle était même trop réaliste et vivante (chansons № 15, 16 et 17), sans avoir passé au préalable des filtres d'embellissement de l'image. Dans certaines chansons cette image est plus détaillée, car leurs auteurs voulaient accentuer l'image du confinement dans différentes pièces d'une maison ou d'un appartement (chansons № 1 et 4). Parfois, la caméra est changée d'endroits pour montrer comment les artistes vivent «sans public» dans toutes les scènes de la vie contemporaine (chanson № 12). Il y avait aussi des artistes moins créatifs qui se sont enregistrés dans un seul endroit (chansons № 2 et 5). Nous avons déjà dit plus haut que même la plateforme Zoom a aussi été exploitée dans le montage de certains clips vidéo. Ce qui est le plus important c'est que cette nouvelle esthétique visuelle s'éloigne de l'esthétique que les chansons populaires américaines nous imposent dans les médias visuels, surtout en Serbie. Avec ces exemples les apprenants serbophones vont avoir l'impression d'être dans le contexte de la France et de certains pays 
francophones tels qu'ils sont, d'une manière réelle, simple, stimulante et parfois volontairement provocatrice, car elles nous permettent aussi de voyager dans différents pays francophones.

\subsection{L'humour}

Une attention particulière est portée sur les chansons qui traitent avec une certaine parodie le confinement et la crise sanitaire. Dans chaque situation de crise l'humour, qu'il soit bienveillant ou noir, est le meilleur remède et un encouragement pour tous. Nous allons nous apercevoir que nous ne sommes pas seuls dans ces nouvelles circonstances et nous nous en sortirons mieux.

La parodie dans certaines chansons (№ 3, 4, 5, 11, 12 et 13) nous aide à surmonter le chagrin, le stress, la peur et l'incertitude subie en pandémie. Parfois cela peut être très touchant aussi, et c'est pour cela qu'il faudra bien choisir le contexte et le public avec lequel nous allons exploiter ces chansons, parce que ce qui est drôle et sympathique pour une personne, ne le sera pas obligatoirement pour une autre. Somme toute, ce qui est commun dans cette pandémie c'est la réunion de différents peuples qui voient sous un même angle cette crise, comme une résistance de l'humanité souffrante, et qui dans la plupart des cas apportent une sorte d'optimisme, de sortie, de cette crise sanitaire. "Cette fonction de rêve collectif, d'indispensable évasion, la chanson populaire aujourd'hui l'assume plus quotidiennement que le cinéma et le roman. » (Bernard 1964: 171)

\section{ASPECT LINGUISTIQUE}

En parlant de l'aspect linguistique nous pouvons dire que notre corpus représente un riche trésor linguistique, divers et varié. Toutes les chansons sont favorables pour l'apprentissage/enseignement du FLE tout d'abord grâce à leur authenticité ; la langue réelle et parlée dans un contexte francophone. « La vie, ses vices et sa laideur; le travail et sa monotonie, la masse et son conformisme, les grands problèmes internationaux et l'angoisse qui en résulte peuvent devenir des sujets de chansons ». (Bernard 1964: 172)

À part les différents niveaux de langue (familier, standard et vulgaire), chacune est riche d'un nouveau vocabulaire qui concerne la pandémie et le monde en confinement (la COVID-19, les gestes barrières, le rereconfinement, etc.). Certains artistes se sont aussi servis de jeux de mots dans les titres ou dans les paroles des chansons, tout en exploitant un air connu, où on chante l'actualité du monde en pandémie dans un sens parodique (№ 8 « On a fermé tous les cinés » (Le 
Livre de la Jungle), № 9 «En confinement (La Petite Sirène) », № 10 «Plus de fête » (La Belle et La Bête) », № 11 «Souvenir de confinement - La Covid Fringante » des Cowboys Fringants, № 12 «Sans public», Parodie Georges Brassens «Les amoureux des bancs publics », № 13 Pierre Perret «Les confinis »). Parmi notre corpus seulement trois chansons n’ont pas été sous-titrées (chansons № $2,7^{7}$ et 14 ), et toutes les autres sont accompagnées de paroles, certaines ont même des sous-titres en anglais (chanson № 6). Les chansons qui n’ont pas de paroles comme accompagnement visuel sont celles qui sont déjà reprises sous-titrées (chansons № 7 et 17), où simplement n’en possèdent pas (chansons № 2 et 14). L'objectif des sous-titres en pandémie est de communiquer à tous un même message transparent et de s'assurer de la bonne compréhension, car il peut s'agir aussi de variétés lexicales du français parlé dans différents pays francophones, où bien d'un accent un peu fort, du français parlé dans différentes régions françaises (chansons № 1, 3, 4, 5, 6, 8, 9, 10, 11, 12, 13, 15 et 16).

\section{QUELQUES PISTES D'EXPLOITATION DES CHANSONS EN CLASSE DE FLE}

«Parmi la diversité de documents authentiques dont peut disposer le professeur de FLE, la chanson est l'un des outils les plus utilisés par les enseignants ». (Boza Araya 2012: 1) Cependant, à son origine elle «n'était pas prévue pour un usage pédagogique. Aujourd'hui, dans les méthodes communicatives il y a une tendance à l'introduire de plus en plus [...].» (ManićMatić, Popović 2012: 355).

Les chansons de notre corpus sont avantageuses pour plusieurs raisons. Tout d'abord elles sont toutes facilement accessibles sur la plateforme YouTube. En tant que supports pédagogiques actuels et authentiques, elles permettent aux apprenants de se sensibiliser au contexte réel, non seulement français, mais aussi francophone. Grâce à leurs formes courtes, visuelles et sonores, elles représentent des documents favorables et attrayants pour un cours d'une durée de 45 minutes $^{8}$, et dans le contexte universitaire, double cours, nous pouvons travailler davantage

\footnotetext{
${ }^{7}$ Cette chanson est reprise de la chanson № 6 qui par contre sur YouTube contient les soustitres.

${ }^{8}$ Dans le système éducatif en Serbie (écoles primaires et secondaires), un cours dure 45 minutes dans les circonstances normales, mais en pandémie il est réduit à 30 minutes. Dans le contexte universitaire il s'agit plutôt d'un double cours de 45 minutes, c'est-à-dire de 1 heure 30 minutes.
} 
sur leur exploitation et varier les chansons francophones en tant que supports pédagogiques. De même, ces chansons qui visent un public des niveaux A1-B2, peuvent aussi assurer le travail sur toutes les compétences (linguistique, sociolinguistique et pragmatique (CECRL ${ }^{9}$ 2000: 17-18), ainsi que sur les activités langagières (compréhension écrite et orale/production écrite et orale).

\begin{tabular}{|c|c|c|c|c|c|c|}
\hline $\begin{array}{c}\text { Chanson } \\
\text { № }\end{array}$ & $\begin{array}{c}\text { Traitant } \\
\text { du lexique } \\
\text { de la } \\
\text { pandémie }\end{array}$ & 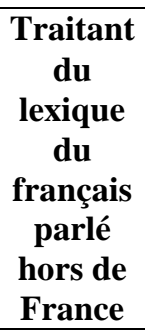 & $\begin{array}{c}\text { Traitant } \\
\text { de } \\
\text { l'humour/ } \\
\text { de la } \\
\text { parodie }\end{array}$ & $\begin{array}{c}\text { Traitant } \\
\text { de } \\
\text { l'humanité }\end{array}$ & $\begin{array}{c}\text { Esthétique } \\
\text { visuelle - } \\
\text { chagement } \\
\text { de } \\
\text { l'intérieur } \\
\text { et/ou de } \\
\text { l'extérieur }\end{array}$ & $\begin{array}{c}\text { Esthétique } \\
\text { musicale } \\
\text { multi- } \\
\text { instrumentiste } \\
(+) / o u \text { la } \\
\text { chanson basée } \\
\text { sur un air } \\
\text { connu } \\
\end{array}$ \\
\hline 1. & + & & + & & + & + \\
\hline 2. & + & & & + & & air connu \\
\hline 3. & + & & + & & & + \\
\hline 4. & + & + & + & & + & air connu \\
\hline 5. & + & & + & & & air connu \\
\hline 6. & + & & & + & + & + \\
\hline 7. & + & + & & + & + & + \\
\hline 8. & + & & + & & + & + \\
\hline 9. & + & & + & & + & + \\
\hline 10. & + & & + & & + & + \\
\hline 11. & + & + & + & & + & air connu \\
\hline 12. & + & + & + & & + & air connu \\
\hline 13. & + & & + & & + & + \\
\hline 14. & + & & & + & + & +/air connu \\
\hline 15. & + & & & + & + & + \\
\hline 16. & + & & & + & + & + \\
\hline 17. & + & & & + & + & + \\
\hline
\end{tabular}

Tableau 1.

Étant donné leur côté ludique elles peuvent stimuler une bonne motivation car on les écoute surtout pour le plaisir. "L'utilisation de la langue pour le rêve ou le plaisir est importante au plan éducatif [...]» (CECRL 2000: 47). Cependant, la question qu'on se pose ici est: pour qui elle implique du plaisir? "Pour l'enseignant ou pour l'apprenant? Pour les deux ?»(Gourvennec 2017: 95). Étant

${ }^{9} \mathrm{CECRL}$ - Cadre européen commun de référence pour les langues. 
donné que la chanson ne cesse pas de changer elle représente une découverte pour les deux. «[...] les deux options ont leur intérêt (et qu'on peut évidemment [...] combiner au cours de l'année), mais que chacun implique une réflexion permettant d'évaluer le choix avant de le valider» (Gourvennec 2017: 97).

Nous allons donner un aperçu tabulaire qui va nous aider à mieux choisir une ou des chansons pour travailler différentes compétences (voir Tableau 1 ci-dessous). Bien qu'elles témoignent toutes de la pandémie, on peut quand même en trouver les différents aspects selon lesquels on va définir les objectifs qu'on devrait atteindre en cours de FLE.

Le choix de la chanson dans l'exploitation va dépendre des objectifs qu'un enseignant devra atteindre dans différentes compétences linguistiques (vocabulaire, phonétique, niveaux de langues, variété du français, phonétique, etc.), socioculturelles (pandémie, confinement, gestes barrières, règles différentes, monuments, villes et pays francophones, scène politique, esthétique musicale et visuelle, humour, parodie, etc.) et pragmatique «qui recouvre l'utilisation fonctionnelle des ressources de la langue » (CECRL 2000: 18). Une fois le clip vidéo choisi, on peut passer aux étapes suivantes de l'exploitation pédagogique de la chanson «1. Définir la tâche, 2. Définir les objectifs et compétences visées, 3. Sélectionner et analyser les supports à utiliser, 4. Planifier la tâche, 5. Introduire la tâche, 6. Préparer la tâche, 7. Réaliser la tâche et 8. Évaluer la tâche » (Gourvennec 2017: 180-181). Pour aller plus loin on peut toujours proposer aux apprenants de créer une «playlist» musicale reflétant le monde francophone et ensuite échanger les «playlists » différentes entre les apprenants où ils vont nous faire découvrir leurs goûts musicaux dans une atmosphère sympathique et créative. Cela a aussi un bon impact sur la motivation, non seulement pour les apprenants qui aiment la musique, et pourraient s'en inspirer, mais aussi pour tous les apprenants de FLE qui voudraient se familiariser davantage avec la culture francophone. Cependant, il faudrait aussi avoir en vue un certain désavantage de l'exploitation des clips vidéo «dans la mesure où ils incitent parfois à la passivité le spectateur qui se laisse bercer par les images sans vraiment y réagir » (Defays 2018: 308). Dans ces cas-là, il revient à l'enseignant d' '« encourager ses apprenants à une écoute active, voire critique, et sélectionner, de préférence, des extraits courts qui permettent de maintenir leur attention éveillée » (Defays 2018: 308).

\section{CONCLUSION}

La nouvelle réalité en temps de pandémie a fait ressortir de nouveaux phénomènes socioculturels et la chanson n'a pas été épargnée. Dans notre contexte de pandémie, elle nous offre une autre perception du langage dans un contexte artistique. 
De même elle témoigne de solidarité et d'universalité d'une humanité unie contre la pandémie.

Pour pouvoir être comprise dans le monde entier elle a été sous-titrée, chantée dans d'autres langues, et même traduite en lingua franca, en anglais. Elle est devenue un appel citoyen pour une vie normale dans la crise sanitaire de par sa forme internationale, presque hymnique. Grâce aux réseaux sociaux elle circule vite et elle est accessible partout facilement. Les chanteurs ont trouvé un autre moyen de se manifester, de s'exprimer, de faire parfois appel à leurs congénères et de se révolter contre les restrictions comportementales prescrites par les gouvernements et les institutions sanitaires partout dans le monde. La chanson est l'un des outils avec laquelle ils expriment globalement leur mécontentement, essayent de lutter tout en éveillant la conscience des autres gens, ou encore chantent sur de nouvelles conditions avec un humour bienveillant ou parfois noir. Elle nous permet de vivre les cultures. Sa forme visuelle et musicale, ainsi que sa durée, font d'elle un excellent support pédagogique en classe de FLE. Sa forme langagière, avec toutes ses variétés et contextes socioculturels réels francophones, pourrait influencer davantage la motivation dans l'apprentissage/enseignement du FLE. De plus, en pandémie elle efface les frontières entre les peuples car nous sommes tous pareillement concernés par la crise globale. De cette façon elle nous encourage et transmet une sorte d'optimisme. Par ailleurs, sa mélodie ne peut qu'être bénéfique pour la santé mentale et ses paroles contribuent largement à l'éveil de la conscience humaine en nous rassurant qu'on n'est pas seul dans cette crise sanitaire globale. Nous sommes d'avis qu'il est toujours important d'encourager et rassurer les apprenants, surtout dans les crises, de tenter de s'adapter aux nouvelles circonstances, de ne pas arrêter d'apprendre, de s'enrichir, et de partager les bonnes valeurs de la citoyenneté mondiale.

\section{CORPUS}

Les Goguettes (en trio mais à quatre), "T'as voulu voir le salon », source URL consultée le 21 avril 2020 : <https://www.youtube.com/watch?v= BFOJtRFIY-8>.

Johnny Boy, «Chanson pour la pandémie », source URL consultée le 1 avril 2020 : <https://www.youtube.com/watch?v=wvoMxVqvg88>.

Sandrine Sarroche, «Elle vendait des attestations et aussi ... », source URL consultée le 26 mars 2021 : <https://www.facebook.com/watch/?v= $1750699935102471>$.

Danick Martineau, «Corona Virus », source URL consultée le16 mars 2020 : <https://www.youtube.com/watch?v=O7W8j-hBP94>. 
Alex Fredo, «Lundi matin - PARODIE DU RECONFINEMENT », source URL consultée le 12 novembre 2020 : <https://www.youtube.com/watch?v=_ 97SBRGHRAM>.

Symphonie confinée «La tendresse », source URL consultée le 29 mars 2020 : $\langle$ https://www.youtube.com/watch?v=rEjvRktXeis>.

«La tendresse », Reprise de la chanson № 6, Chorale virtuelle du Maroc - Les choristes des Écoles Catholiques de Mohammedia, source URL consultee le 12 mai 2020 : 〈https://www.youtube.com/watch?v=ISfv54nczNE〉.

PARODIE COVID : «On a fermé tous les cinés » (Le Livre de la Jungle), source URL consultée le 14 décembre 2020 : <https://www.youtube.com/watch? $\mathrm{v}=\mathrm{bbc} 2 \mathrm{u} 5 \mathrm{kd} 1 \mathrm{XI}>$.

PARODIE COVID : «En confinement (La Petite Sirène) », source URL consultée le 26 novembre 2020 : <https://www.youtube.com/watch?v=HDeXUfY mGtw>.

PARODIE COVID : «Plus de fête » (La Belle et La Bête)», source URL consultée le 26 octobre 2020: <https://www.youtube.com/watch?v= SUGijS5USWw\&t=33s>.

SOUVENIR DE CONFINEMENT - LA COVID FRINGANTE, source URL consultée le 9 octobre 2020: <https://www.youtube.com/watch?v= 13sxuiiMLm4>.12. « Sans public », Parodie

Georges Brassens «Les amoureux des bancs publics », source URL consultée le 15 mars $2020:$ https://www.youtube.com/watch?v=qVUxPC8qfsQ>.

Pierre Perret «Les confinis», source URL consultée le 9 juin 2020 : $\langle$ https://www.youtube.com/watch?v=zA2JjodD6IU>.

Gilles Hudon, « Nous sommes le monde (We are the World) », source URL consultée le 10 avril 2020 : <https://www.youtube.com/watch?v=2LIcu-bioo>.

«HK (Kaddour Hadadi) - On veut continuer à danser encore !», source URL consultée le 26 décembre $2020:$ https://www.youtube.com/watch?v= g48_U2T3QRE>.

«DANSER ENCORE - Flashmob - Gare du Nord - 4 Mars $2021 »$, Reprise de la chanson № 15, source URL consultée le 5 mars 2021 : <https://www.youtube.com/watch?v=Gq9qFvoMKaY>.

«DANSER ENCORE de HK (Kaddour Hadadi)», les reprises les plus populaires, source URL consultée le 21 avril 2021 : <https://www.facebook.com/ humanite.fr/videos/3092324091025735>. 


\section{BIBLIOGRAPHIE ET SITOGRAPHIE}

Bernard, Y. (1964). La chanson, phénomène social, in Revue française de sociologie: 166-174; source URL consultée le 27 avril 2021 : <www.persee.fr/doc/rfsoc_0035-2969_1964_num_5_2_6324>.

Boza Araya, V. (2012). La chanson et son enseignement - Quelles stratégies dans la classe FLE ? Pour quels publics ?, in Revista de Lenguas Modernas 16: 197-213. https://revistas.ucr.ac.cr/index.php/rlm/article/view/12605

CECRL - Cadre européen commun de référence pour les langues (2000). Source URL consultée le 2 avril 2021 : <https://www.coe.int/fr/web/commoneuropean-framework-reference-languages $>$.

Defays, J.-M. (2018). Enseigner le français langue étrangère et seconde. Bruxelles : Éditions Mardaga.

Gourvennec, L. (2017). Paroles et musique : le français par la chanson. Paris : Hacchette.

Knežević, S. (2011). Muzička estetika i instrumenti. Sombor: Gradska biblioteka „Karlo Bijelicki”; Gradski muzej Sombor.

Manić-Matić, V., Popović N. (2012). La chanson francophone en classe de FLE, in Jezici $i$ kulture $u$ vremenu $i$ prostoru 1, ur. S. Gudurić (Novi Sad: Filozofski fakultet): 355-361.

Zarka, Y. C. (2004). Cités 19 : Que dit la chanson ? Paris : PUF.

Source URL consultée le 10 mars 2021 : <https://www.youtube.com/watch?v= g3NT0cirYh0>.

Source URL consultée le 21 avril 2021 : <https://www.facebook.com/RFI/videos/ 159376889330430>.

Source URL consultée le 21 avril 2021 :

$<$ https://actu.fr/normandie/cherbourg-en-cotentin_50129/le-chanteur-hk-et-sonspectacle-revendicatif-danser-encore-bientot-a-cherbourg_42103558.html? utm_medium=Social\&utm_source=Facebook\#Echobox $=1622112695>$. 
Vanja Manić-Matić

Univerzitet u Novom Sadu

Filozofski fakultet, Odsek za romanistiku

\author{
FRANKOFONA PESMA U DOBA PANDEMIJE - \\ RAZMIŠLJANJA O STANJU UMETNOSTI I UTICAJ U UČENJU/NASTAVI \\ FRANCUSKOG KAO STRANOG JEZIKA
}

\title{
Sažetak
}

Živimo u novoj eri koju su oblikovali pandemija i širenje virusa COVID-19. Kao rezultat toga nametnuta su nam nova pravila i mnoga ograničenja u svim oblastima svakodnevnog života, pa tako i u muzici. Od proglašenja pandemije, umetnici širom sveta pokušavaju da istupe pred ovom novom stvarnošću i da se bore za bolje uslove rada i svoje živote uopšte. U radu ćemo se fokusirati na korpus od sedamnaest francuskih pesama koje su odjeknule internetom i pokazaćemo kakav uticaj imaju na čovečanstvo i kako se mogu koristiti u učenju/nastavi francuskog kao stranog jezika. Na osnovu analitičke i komparativne metodologije pokazaćemo koje su prednosti njihove upotrebe na časovima francuskog i šta nam one donose na tehničkom, sociokulturološkom i jezičkom nivou. Verujemo da je na nastavnicima da prate svaku sociokulturološku promenu, prilagođavaju je i dalje integrišu ne samo u učenje/nastavu jezika već i u učenje/nastavu uopšte.

Ključne reči: pandemija, pesma, francuski, frankofonija, tehnologija, sociokulturalnost, lingvistika, nastava/ učenje, francuski kao strani jezik.

Article reçu: le 5 mai 2021

Article accepté: le 19 juillet 2021 
